\title{
Cardiac $\mathrm{AT}_{1}$ Receptor-Dependent and IGF1 Receptor-Independent Signaling Is Activated by a Single Bout of Resistance Exercise
}

\author{
S. F. S. MELO ${ }^{1,2}$, V. G. BARAUNA ${ }^{2}$, T. FERNANDES ${ }^{1}$, E. C. CARMO ${ }^{1}$, \\ C. R. O. CARVALHO ${ }^{3}$, E. M. OLIVEIRA ${ }^{1}$
}

${ }^{1}$ Laboratory of Biochemistry and Molecular Biology of the Exercise, Department of Human Movement Biodynamics, School of Physical Education and Sport University of São Paulo, São Paulo, Brazil, ${ }^{2}$ Laboratory of Molecular Physiology, Health Sciences Center, Federal University of Espírito Santo, Vitória, Brazil, ${ }^{3}$ Laboratory of Cellular Signaling, Department of Physiology and Biophysics, University of São Paulo, São Paulo, Brazil

Received December 2, 2016

Accepted January 27, 2017

On-line September 22, 2017

\section{Summary}

$\mathrm{AT}_{1}$ receptor (AT1R) blockade prevents physiological cardiac hypertrophy induced by resistance training. Also, our group showed that a single bout of resistance exercise (RE) activates the AKT/mTOR which was also inhibited by AT1R blocker. Here, we investigated whether IGF1-receptor (IGF1-R) and MAPKs were also activated after a single bout of RE. Wistar rats were divided into Sedentary (Sed), Sedentary treated with losartan (Sed+LOS), Exercise (EX), and Exercise treated with losartan (EX+LOS). Cardiac tissue was obtained 5 and $30 \mathrm{~min}$ after 4 sets of 12 repetitions of squat exercise ( $80 \% 1 \mathrm{RM})$. We demonstrated that a single bout of RE did not induce IGF1-R tyrosine phosphorylation. ERK1/2 and P38 phosphorylation levels were elevated in the EX $5 \mathrm{~min}$ and EX 30min groups however, only ERK1/2 was inhibited by losartan treatment (AT1R blocker). Next, we showed that $\beta$-arrestin-2 expression increased $28 \%$ in trained animals compared to sedentary group. Altogether, our results demonstrate that $A T 1 R$, but not IGF1-R, may exert the hypertrophic cardiac stimulus RE-induced. Also, activation of AKT/mTOR and ERK1/2 pathways may occur through the $\beta$-arrestin-dependent pathway.

\section{Key words}

$\mathrm{AT}_{1}$ receptor - Cardiac hypertrophy • $\beta$-arrestin - Resistance exercise

\section{Corresponding author}

E. M. Oliveira, Laboratory of Biochemistry and Molecular Biology of the Exercise, School of Physical Education and Sport, Av. Prof. Mello Moraes, 65, Cidade Universitária, 05508-9000, São Paulo, SP, Brazil. Fax: 55-11-3813-5921. E-mail: edilamar@usp.br

The cardiac hypertrophy $(\mathrm{CH})$ is a well-known response to increased hemodynamic load to the heart and may be induced by different factors such as mechanical stress or neurohumoral factors (Iemitsu et al. 2006). $\mathrm{CH}$ can be classified as pathological or physiological. The pathological $\mathrm{CH}$ occurs in response to situations such as hypertension, valve disease, myocardial infarction or genetic mutations. On the contrary, physiological $\mathrm{CH}$ occurs during the normal growth of the heart from embryonic and fetal stages of development until the adult life and also in response to exercise training (Fernandes et al. 2015).

It is well established that angiotensin II type 1 receptor (AT1R) plays an important role in the development of pathological $\mathrm{CH}$. However, we have showed increased AT1R expression after exercise training (swimming training) (Oliveira et al. 2009), and AT1R blockade has prevented physiological $\mathrm{CH}$ induced by exercise training (Barauna et al. 2008).

It is generally accepted that activation of G-protein pathway of the AT1R leads to pathological 
cardiac remodeling, while G-protein independent pathways, mainly thought $\beta$-arrestins, increase cardiac performance, diminish cardiac fibrosis and decreases cardiomyocytes apoptosis (Violin et al. 2014). We have showed that a single bout of resistance exercise (squat exercise) activates the well-known hypertrophic pathway AKT/mTOR pathway thought AT1R in the heart (Melo et al. 2011). However, although the IGF1-R has already been described to be activated by aerobic training (Kemi et al. 2008), it is still unclear its role in response to resistance training.

To further dissect the role of AT1R in the physiological $\mathrm{CH}$ exercise-induces, we aimed to verify the AT1R intracellular pathways MAPKs and $\beta$-arrestin as well as its crosstalk with IGF1-R in response to resistance training.

Thirty-six male Wistar rats (10-week-old) were randomly divided into six groups ( $\mathrm{n}=6$ /group): sedentary control (Sed); sedentary control treated with losartan (SED+LOS); exercised and killed after 5 min (EX 5min); exercised and killed after $30 \mathrm{~min}$ (EX 30min); exercised treated with losartan and killed after 5 min $(\mathrm{EX}+\mathrm{LOS}$ $5 \mathrm{~min}$ ) and exercised treated with losartan and killed after $30 \mathrm{~min}$ (EX+LOS 30min). Losartan treatment $\left(20 \mathrm{mg} \cdot \mathrm{kg}^{-1} \cdot \mathrm{day}^{-1}\right)$ was administered in drinking water (Oliveira et al. 2009, Barauna et al. 2008). The treatment began one week before the exercise protocol. In the chronic protocol, twelve male Wistar rats (10-week-old) were randomly divided into two groups ( $n=6 /$ group): sedentary and trained. Animals were housed in standard cages, with food and water ad libitum. All the protocols and surgical procedures were in accordance with the guidelines of the Brazilian College for Animal Experimentation and were approved by the Ethics Committee of the Institute of Biomedical Science of the University of São Paulo.

Animals were acutely ( 1 exercise session) and chronically ( 8 weeks protocol) exercised as previously described by our group (Barauna et al. 2008, Melo et al. 2015, Melo et al. 2011). Rats fitted with canvas jackets were able to regulate the twisting and flexion of their torsos and were suspended in a standard position on their hind limbs. An electrical stimulation $(20 \mathrm{~V}, 0.3-\mathrm{s}$ duration, at 3-s intervals) was applied to the rat's tail by means of a surface electrode. As a result, the rats flexed their legs repeatedly, which lifted the weight arm of the training apparatus. The exercise groups performed 4 sets of 12 repetitions, with a 90-s rest period between each set and an overload of $80 \%$ of $1 \mathrm{RM}$. This exercise regimen was performed after one week of adaptation to the apparatus. In the acute protocol animals performed a single bout of resistance exercise and rats were killed by decapitation after 5 or $30 \mathrm{~min}$ of completion the exercise and its heart were removed. In the chronic protocol animals were trained by resistance training for eight weeks, 5 times per week as described above.

The frozen LVs pieces were homogenized in cell lysis buffer (100 mM Tris, $50 \mathrm{mM} \mathrm{NaCl}, 10 \mathrm{mM}$ EDTA, $1 \%$ Triton X-100 and protease inhibitors. Insoluble material was removed by centrifugation. The supernatant was used for immunoprecipitation with anti-IGF1-Ras described elsewhere. Western blot was performed as previous published by our group (Melo et al. 2015, Barauna et al. 2008, Melo et al. 2011) then probed with antibody directed against ERK1/2, P38 and JNK, as well as the corresponding phosphorylated proteins $\mathrm{p}$-ERK1/2, p-P38 and p-JNK (1:1000; Abcam, Cambridge, UK). Total protein expression was used for normalization of the results.

Frozen LVs pieces were homogenized in trizol and RNA was isolated according to the manufacturer's instructions (Invitrogen Life Technologies, Strathclyde, UK). $\beta$-arrestin-2 mRNA expression was assessed by oligonucleotides primers as follows: 5'-GGACGTTGA CATTGAGGGGT-3' and 5'-GGACGTTGACATTGA GGGGT-3'. Cyclophilin mRNA expression (5'-TGG CAAGCATGTTGGGTCTTTGGGAG-3' and 5'-GGT GATCTTCTTGCTGGTCTGCCATTC-3') was measured as internal control. Quantification of the target genes expression was performed with a SYBR green PCR Master Mix (Applied Biosystems, Foster City, CA, USA). The relative expression of the mRNA was performed by real-time PCR in the ABI PRISM 7500 Sequence Detection System (Applied Biosystems, Foster City, USA).

Differences between groups (trained vs. sedentary) were assessed using unpaired t-tests. Comparison between groups (with or without losartan treatment vs. sedentary, exercise $5 \mathrm{~min}$ or exercise $30 \mathrm{~min}$ ) were accomplished by two-way ANOVA. Tukey HSD post-hoc test was employed for comparison among mean values, when ANOVA indicated significant changes. $\mathrm{P}<0.05$ was accepted as statistically significant. All results are presented as mean \pm standard error of the mean (SEM).

Figure 1 depicts the Western blot results of the MAPK family members, ERK1/2, P38, and JNK proteins in the rat LV. Phosphorylation of ERK1/2 increased in 
the EX 5min (26\%) and EX 30min (59\%) groups, respectively, compared with the sedentary control while losartan inhibited this increase in both groups (Fig. 1A). There was a small, but significant, elevation in P38 phosphorylation in both EX 5min (16\%) and EX 30min (14\%) that was not inhibited by losartan (Fig. 1B). There was no difference in the JNK activation among groups (Fig. 1C). Similarly, Iemitsu et al. (2006) investigated the activation of MAPKs in hearts of rats submitted to aerobic training and also reported increase in P38 and ERK1/2 phosphorylationafter 30-min of exercise. The data from our study show that increase in the phosphorylation of ERK1/2 is mediated by AT1R since losartan abrogated this increase. On the other hand, increased P38 phosphorylation was not blocked by AT1R antagonist. Still, it is unclear the linking pathway between AT1R and ERK1/2.

Previous studies showed that the IGF1-R is involved in cardiomyocytes hypertrophy in both elite athletes and trained rats (Scheinowitz et al. 2003).
Activation of the IGF1-R is associated with the $\mathrm{PI} 3 \mathrm{~K} / \mathrm{AKT} / \mathrm{mTOR}$ pathway when submitted to aerobic training (DeBosch et al. 2006). Since we have already showed AKT/mTOR activation by resistance training (AKT phosphorylation increased $60 \%$ in both the EX 5min and EX 30min groups; mTOR phosphorylation increased $21 \%$ and $65 \%$ in the EX $5 \mathrm{~min}$ and EX 30min groups, respectively. Both activations were inhibited by losartan (Melo et al. 2011), we next investigated the IGF1-R activation status. Figure 1D shows the IGF1-R phosphorylation level after a single bout of resistance exercise. IGF1-R was immunoprecipitated from LV and subjected to immunoblotting with antiphosphotyrosine antibody. There were no differences in tyrosine phosphorylation levels of IGF1-R. Still, it is also unclear the linking pathway between AT1R and AKT/mTOR.

In summary, the data showed here and previous published by us show that AT1R participates in the activation of both AKT/mTOR and ERK1/2 following a single bout of resistance exercise. AT1R has been
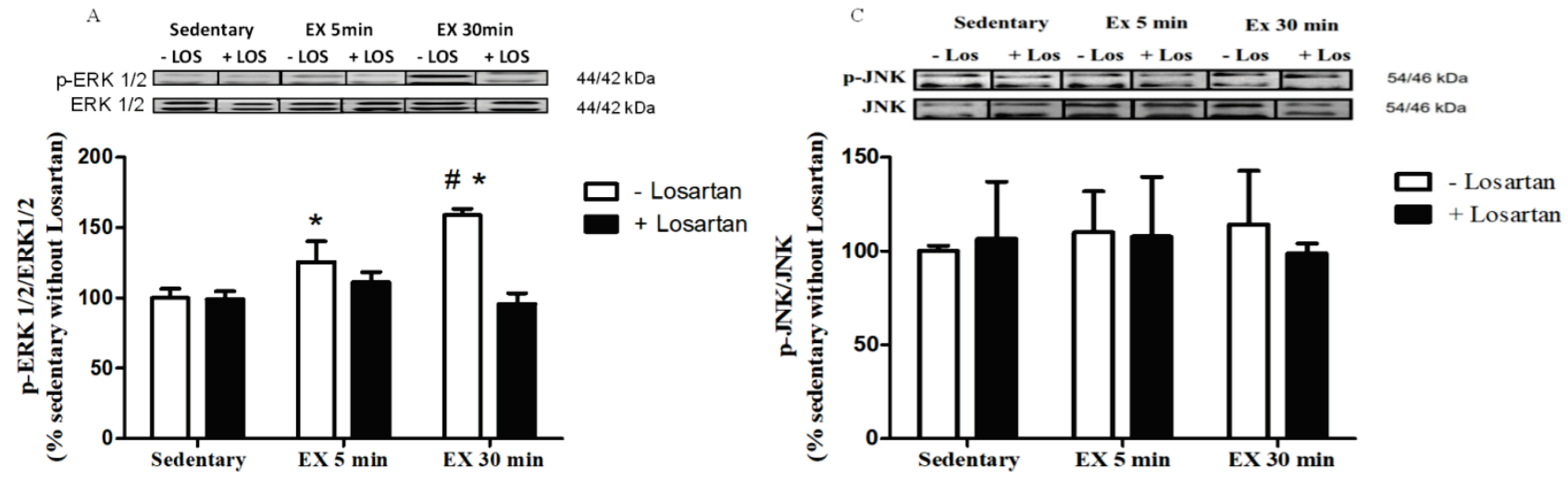

B

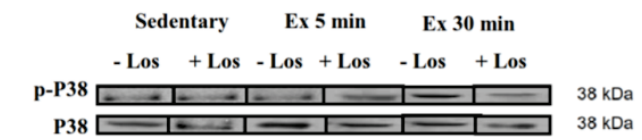

$\mathrm{D}$

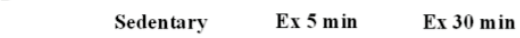

$-\operatorname{Los}+\operatorname{Los}-\operatorname{Los}+\operatorname{Los}-\operatorname{Los}+\operatorname{Los}$
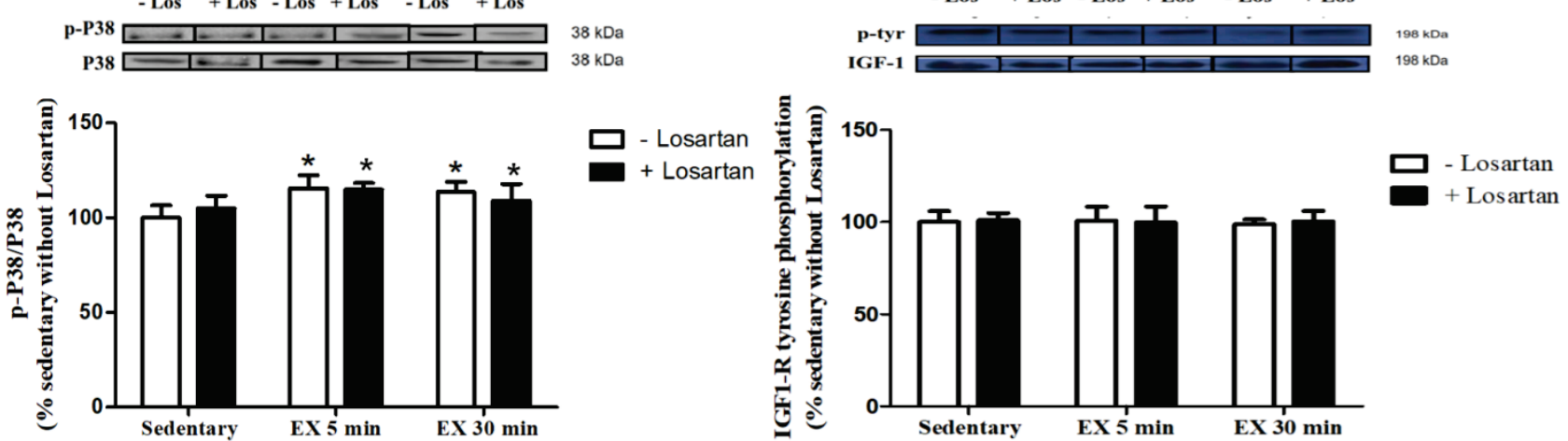

Fig. 1. MAPKs and IGF1-R activation by resistance training. Effect of a single bout of resistance exercise on the phosphorylation-to-total protein ratio of the MAPK family members and tyrosine phosphorylation of IGF1-R in rat left ventricles (A) ERK1/2 phosphorylation, (B) p38 phosphorylation, (C) JNK phosphorylation, and (D) IGF1-R phosphorylation. Data are reported as mean \pm SEM, $n=6 / g r o u p$. * $\mathrm{P}<0.05$ vs. Sed group. ${ }^{*} \mathrm{P}<0.05$ vs. EX+LOS 30min group. Sed - Sedentary group without Losartan. 
shown to regulate both ERK1/2 and AKT in arrestin signalsomes (Kendall et al. 2014). They observed that AT1R promoted cell growth and hypertrophy through $\beta$-arrestin-2-mediated mechanisms. To test whether $\beta$-arrestin-2 may participate in the physiological $\mathrm{CH}$ exercise training-induces, rats were subjected to resistance training for eight weeks. We have observed $\mathrm{CH}$ of $22 \%$ in the left ventricular mass/body weight ratio (Melo et al. 2015) and Figure 2 shows that $\beta$-arrestin-2 gene expression increased by $28 \%$.

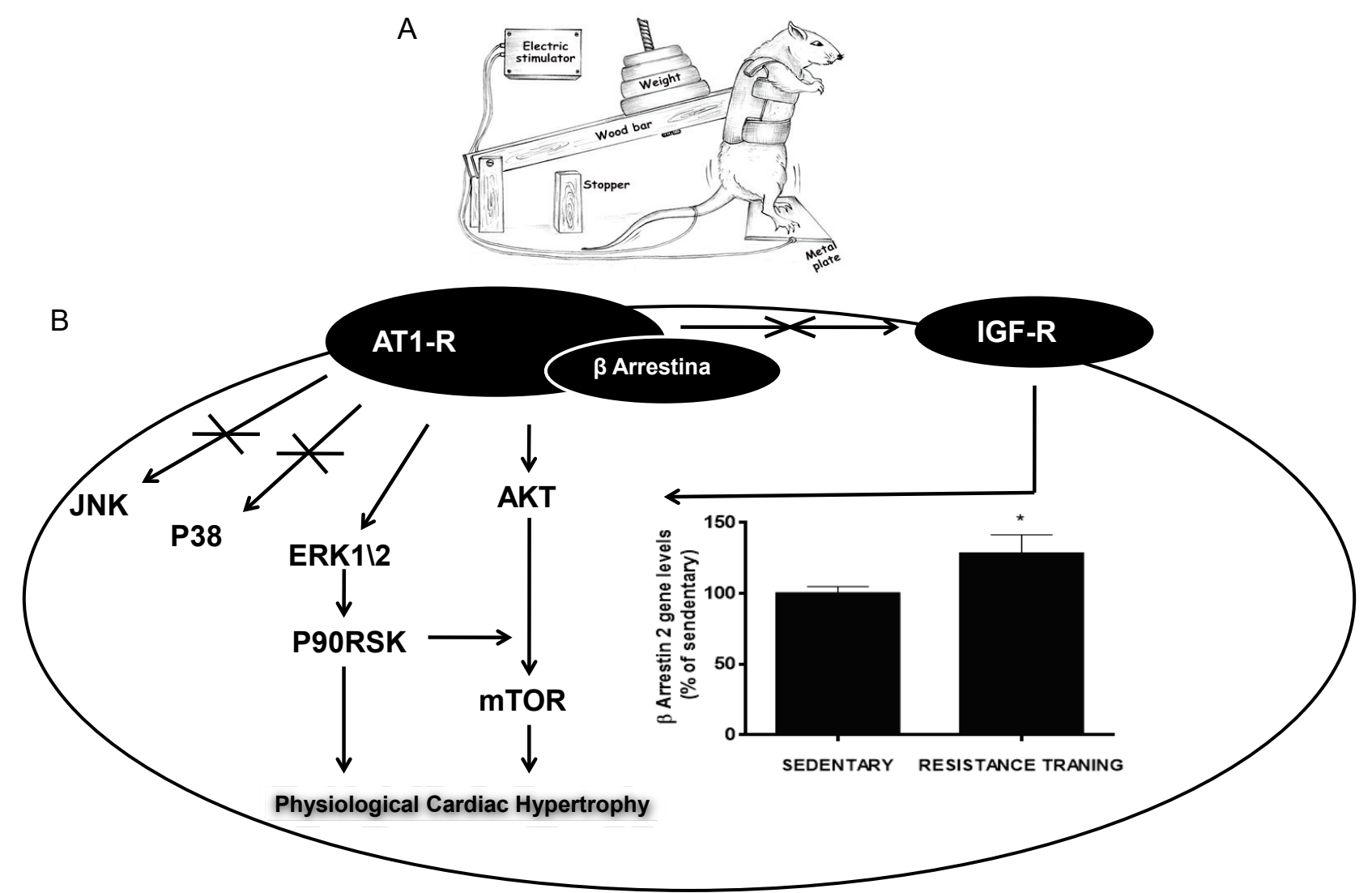

Fig. 2. Schematic summary of the AT1R signaling after a single bout of resistance exercise. (A) Squat-training apparatus used to perform resistance exercise in rats (Melo et al. 2015). (B) $\beta$-arrestin-2 mRNA expression after 8 weeks of resistance training. Data are reported as mean $\pm \mathrm{SEM}, \mathrm{n}=6 /$ group. $* \mathrm{P}<0.05$ vs. Sed group. Sed - Sedentary group without Losartan.

Altogether, we have studied the cardiac intracellular signaling in response to resistance training. Interestingly, we demonstrated that a single bout of resistance exercise does not induce IGF1-R tyrosine phosphorylation, which excludes a possible AT1R and IGF1-R crosstalk. Also, we suggest that ERK1/2 and AKT/mTOR activation by resistance training, but not $\mathrm{p} 38$ and JNK, may occur by the AT1R $\beta$-arrestin-dependent pathway (Fig. 2). Our results agree with the dual function of the AT1R both in physiological and pathological cardiac remodeling which depends on the intracellular signaling activated. AT1R may have beneficial effects when it is biased activated to the $\beta$-arrestin signaling by mechanical forces (Rakesh et al. 2010, Barauna et al. 2013), exercise training (Barauna et al. 2008) and biased ligands (TRV or SII) (Violin et al. 2014). The present study helps to understand the molecular mechanisms responsible for mediating the different forms of cardiac hypertrophy with exercise.

\section{Conflict of Interest}

There is no conflict of interest.

\section{Acknowledgements}

This work was supported by FAPESP (Project numbers: 07/52457-3; 10/09438-0) and FAPES (Project numbers: 1018/2015 and 67659551/2015). E. M. Oliveira was recipient of a CNPq Fellowship (No. 308267/2013-3) and V. G. Barauna CNPq-Universal (No. 485873/2012-5). CAPES-Proex. 


\section{References}

BARAUNA VG, MAGALHAES FC, KRIEGER JE, OLIVEIRA EM: AT1 receptor participates in the cardiac hypertrophy induced by resistance training in rats. Am J Physiol Regul Integr Comp Physiol 295: 381-387, 2008.

BARAUNA VG, MAGALHAES FC, CAMPOS LCG, REIS RI, KUNAPULI SP, COSTA-NETO CM, MIYAKAWA AA, KRIEGER JE: Shear stress-induced Ang II AT1 receptor activation: G-protein dependent and independent mechanisms. Biochem Biophys Res Commun 434: 647-652, 2013.

DEBOSCH B, TRESKOV I, LUPU TS, WEINHEIMER C, KOVACS A, COURTOIS M, MUSLIN AJ: Akt1 is required for physiological cardiac growth. Circulation 113: 2097-2104, 2006.

FERNANDES T, BARAÚNA VG, NEGRÃO CE, PHILLIPS MI, OLIVEIRA EM: Aerobic exercise training promotes physiological cardiac remodeling involving a set of microRNAs. Am J Physiol Heart Circ Physiol 309: H543-H552, 2015.

IEMITSU M, MAEDA S, JESMIN S, OTSUKI T, KASUYA Y, MIYAUCHI T: Activation pattern of MAPK signaling in the hearts of trained and untrained rats following a single bout of exercise. J Appl Physiol 101: 151-163, 2006.

KEMI OJ, CECI M, WISLOFF U, GRIMALDI S, GALLO P, SMITH GL, CONDORELLI G, ELLINGSEN O: Activation or inactivation of cardiac Akt/mTOR signaling diverges physiological from pathological hypertrophy. J Cell Physiol 214: 316-321, 2008.

KENDALL RT, LEE MH, PLEASANT DL, ROBINSON K, KUPPUSWAMY D, MCDERMOTT PJ, LUTTRELL LM: Arrestin-dependent angiotensin AT1 receptor signaling regulates Akt and mTor-mediated protein synthesis. J Biol Chem 289: 26155-26166, 2014.

MELO SFS, AMADEU MA, MAGALHÃES FC, FERNANDES T, CARMO EC, BARRETTI DLM, BRUM PC, OLIVEIRA EM: Activation of AKT-mTor signaling pathways by angiotensin II receptor type 1 after a session of strength exercise in cardiac muscle of rats. Brazil J Phys Educ Sport 25: 377-385, 2011.

MELO SFS, BARAUNA VG, CARNEIRO MA JR, BOZI LHM, DRUMMOND LR, NATALI AJ, DE OLIVEIRA EM: Resistance training regulates cardiac function through modulation of miRNA-214. Int J Mol Sci 16: 6855-6867, 2015.

OLIVEIRA EM, SASAKI MS, CERÊNCIO M, BARAÚNA VG, KRIEGER JE: Local renin-angiotensin system regulates left ventricular hypertrophy induced by swimming training independent of circulating renin: a pharmacological study. J Renin Angiotensin Aldosterone Syst 10: 15-23, 2009.

RAKESH K, YOO B, KIM I, SALAZAR N, KIM K, ROCKMAN HA: Beta-Arrestin-biased agonism of the angiotensin receptor induced by mechanical stress. Sci Signal 3: 46, 2010.

SCHEINOWITZ M, KESSLER-ICEKSON G, FREIMANN S, ZIMMERMANN R, SCHAPER W, GOLOMB E, SAVION N, ELDAR M: Short- and long-term swimming exercise training increases myocardial insulin-like growth factor-I gene expression. Growth Horm IGF Res 13: 19-25, 2003.

VIOLIN JD, SOERGEL DG, LARK MW: Beta-arrestin-biased ligands at the AT1R: a novel approach to the treatment of acute heart failure. Drug Discov Today Ther Strateg 9: 1-6, 2014. 